\title{
La francophonie post-soviétique. Sa littérature, son cinéma
}

Iulian TOMA, Université Brock

\section{Une posture transculturelle décomplexée}

À une époque où les discours sur la mondialisation connaissent une prolifération et une expansion qui débordent largement les limites des débats socioéconomiques, et révèlent de plus en plus l'incidence culturelle et artistique de ce phénomène, l'étude de la francophonie européenne se doit de mieux élucider la diversité des phénomènes dont elle rend compte. Si l'on accepte l'idée que la culture francophone ne saurait en aucun cas se confondre avec la seule culture des pays de langue française (France, Belgique, Suisse, Luxembourg), qu'elle déborde les frontières nationales et les aires linguistiques, il apparaît nettement que l'Europe francophone est faite aussi, et surtout, d'interactions, par le biais du français, entre les nombreuses cultures du continent. Il existe, chose notoire d'ailleurs, une longue histoire des contacts entre les cultures des pays de langue française, de la France en particulier, et celles des pays européens ayant connu une tradition francophile. Mais cette histoire des interférences francophones à l'intérieur de l'Europe n'est pas linéaire, ni d'autant moins unidimensionnelle. Elle porte l'empreinte au fond des réalités sociopolitiques et culturelles qui façonnent l'histoire européenne des derniers siècles. Pour s'en tenir à l'histoire récente, les conséquences des deux guerres mondiales, les effets des régimes autoritaires qui s'installent dans plusieurs pays d'Europe, ceux de la transition qui s'ensuit, la fondation de l'Union Européenne, le prestige de la culture française, tous ces facteurs majeurs contribuent à modeler au vingtième siècle et au début du vingt-et-unième le visage de la francophonie sur le « vieux continent ».

Ainsi envisagée, la francophonie européenne s'avère plurielle, chacun de ses versants pouvant être exploré séparément. L'un d'entre eux est celui de ce que Joanna Nowicki et Catherine Mayaux appellent «l'autre francophonie », en faisant écho au titre du fameux essai autobiographique Une autre Europe ${ }^{1}$ de Czeslaw Milosz qui désigne par cette formule l'espace culturel de l'Europe Centrale et de l'Est. La prospection de cette branche de la francophonie européenne s'est intensifiée au cours de la dernière décennie ${ }^{2}$, dans la foulée, entre autres facteurs déterminants, de l'essor fulgurant des études francophones classiques (Afrique, Caraibes, Asie). S'imposant ainsi de plus en plus comme une enclave autonome dans le champ 
des dynamiques francophones européennes, les interactions entre les cultures de l'Europe Centrale et Orientale d'une part, et celles des pays francophones occidentaux d'autre part, exigent d'être envisagées davantage dans leur spécificité.

Il est ainsi possible de délimiter, à l'intérieur de l'ensemble composite des phénomènes constituant l'«autre francophonie », un groupe à part dont la formation et la dynamique sont déterminées par la dissolution, à partir de 1989, de l'Union Soviétique et du Bloc de l'Est, car, avec l'effondrement du «Rideau de fer», se met en place sur le continent une nouvelle réalité politique, sociale, institutionnelle et culturelle qui redessine la carte de la francophonie européenne.

Politiquement, les pays libérés de la tutelle soviétique, dans leur grande majorité, se soucient de (re)bâtir leur identité sur la base des principes de la démocratie et du libéralisme. Cette identité est consolidée dans le contexte de l'expansion de l'Union Européenne où certains de ces pays obtiennent le statut d'État-membre (l'Estonie, la Hongrie, la Lettonie, la Lituanie, la Pologne, la Slovaquie, la Slovénie, la République Tchèque en 2004 ; la Roumanie et la Bulgarie en 2007 ; la Croatie en 2013) ou bien se présentent comme candidats à ce statut (l'Albanie, la Macédoine, le Monténégro, la Serbie). Le mot d'ordre devient «intégration », et l'Europe comprend vite les bénéfices, au même titre que les défis, du « communautarisme ».

Toujours pendant leur existence post-soviétique, sur le plan culturel et institutionnel, un nombre important de ces pays deviennent membres de l'Organisation Internationale de la Francophonie (OIF) et adhèrent à l'Agence Universitaire de la Francophonie (AUF). Ainsi multiplient-ils les rapports de collaboration avec des pays francophones de l'Europe occidentale : coopérations et échanges interuniversitaires, traductions d'œuvres marquantes, coproductions cinématographiques, éditions de volumes collectifs, organisation de colloques internationaux, etc. Ce sur quoi repose l'appartenance au monde francophone de ces pays sans locuteurs natifs mais avides de ranimer une tradition culturelle dont ils ont été pendant des décennies coupés, c'est le rapport qu'ils entretiennent avec le français comme langue qui a longtemps porté pour eux les valeurs de l'humanisme occidental.

Socialement, enfin, le dernier quart de siècle est marqué pour les deux hémisphères de l'espace européen par le phénomène de la migration économique dont les bénéfices et impondérables sont partagés et respectivement assumés par les pays concernés. Des centaines de milliers d'immigrants des pays ayant connu le régime communiste s'installent comme résidents 
en France, en Belgique et en Suisse principalement, où ce flux migratoire, quoique moins important que celui d'origine non-européenne (africain et asiatique), n'est pas sans réverbérer sur la perception publique. En ce qui concerne les acteurs principaux de cette dynamique transnationale, ils représentent dans le monde contemporain un groupe d'immigrants distinct qui, tout en se confrontant aux contingences spécifiques de cette forme de mobilité, se rabattent sur leur « européanité » comme refuge contre le dépaysement et antidote aux affres identitaires.

Il y a bien évidemment une ligne de démarcation à tracer dans l'histoire récente de la francophonie européenne dans le secteur des interactions entre les deux grands espaces culturels en question. La chute du mur de Berlin modifie la configuration de ces contacts qui s'intensifient et, qui plus est, modifient leur nature. On ne saurait nier la disparité qui se manifeste du point de vue social, politique et psychologique entre le groupe de ceux qui fuient leur pays sous la pression d'un environnement qu'ils ressentent comme oppressif ou menaçant, et la masse de ceux qui choisissent de résider à l'étranger à la recherche d'une «meilleure vie ». Ce sont là deux types de déplacement, le premier se confondant avec l'exil, le second avec l'émigration. L'un se définit comme impossibilité du retour au pays abandonné, l'autre n'exclut pas l'éventualité de le regagner. À la lumière de cette distinction, il s'impose de constater, en ce qui concerne la mobilité démographique sur l'axe Europe Centrale et Orientale-Europe Occidentale, que l'année 1989 place ce phénomène sur l'orbite de l'immigration en enrayant par là même le cycle de l'exil.

Deux attitudes concourent principalement à modeler la posture transculturelle dans l'espace de la francophonie européenne post-soviétique. D’une part, l'expérience du sujet migrant qui rejoint, après 1989, un pays occidental, afin de s'épargner, dans le sien, les désarrois inhérents à la transition du totalitarisme vers la démocratie et le libéralisme économique, ne se définit pas dans la résistance à l'égard de la culture et de la langue d'accueil. Le discours revendicateur n'est pas le lot de ce groupe culturel francophone. Peu de défis pèsent sur son processus d'acclimatation, et leur genèse n'est ni historiquement déterminée ni de nature religieuse ou d'origine raciale. L'intégration de ce groupe est au contraire facilitée par son européanité. D'autre part, en vertu même de son double ancrage dans l'espace européen, cette nouvelle communauté francophone ne connait généralement le déracinement que sous ses formes bénignes. Les frontières nationales, dans sa représentation de 1'Europe comme référent pluriel, ressemblent plutôt à des portes battantes qu'à des barrières. Certes, le souvenir du mur ayant 
séparé les deux Europe ne s'est pas résorbé, mais il reste latent, dépourvu d'ascendant significatif sur le présent. Aussi peut-on dire qu'à la différence de l'expérience de l'exil fondamentalement liée à la conscience aiguë de la désunion et à l'errance identitaire, la condition d'immigrant dans l'Europe post-soviétique et mondialiste s'ancre dans le terreau du multiculturalisme. Ni manifestement jubilatoire ni affligée, cette posture juxtapose et embrasse des identités culturelles distinctes au lieu d'accuser la dispersion et la perte de l'unité de soi comme cela se produit chez l'exilé. On peut ainsi avancer que le phénomène migratoire déclenché après 1989 en tant que vecteur de la francophonie européenne, pour ne parler que de cet espace culturel, suppose une relation décomplexée avec les facteurs - linguistiques, historiques, identitaires - qui entrent en jeu dans ce processus.

\section{Le discours francophone post-soviétique}

Plusieurs facteurs - sociaux, politiques, culturels - configurent donc la carte de la francophonie européenne du dernier quart de siècle sous l'aspect des interactions entre les deux hémisphères géographiques et culturelles du continent. Peut-on néanmoins parler à l'égard de cette dynamique francophone d'un ressort unificateur qui en ferait un versant autonome de la francophonie européenne ? Peut-on, autrement dit, constater l'existence d'une «francophonie post-soviétique », c'est-à-dire d'un discours émané de ces circonstances historiques qui transporte dans l'espace de la langue française, écrite ou parlée, tout ce qui se pense et s'imagine autour et lors de l'agrégation de cette réalité interculturelle spécifique ?

L'emploi du syntagme discours francophone permet de contourner les apories auxquelles on se confronte lorsqu'on en vient à définir le sens de l'adjectif « francophone » sur le plan des réalités culturelles. On sait désormais, par exemple, que le recours aux critères de la nationalité et de la langue maternelle ne suffit pas pour établir une distinction nette entre auteur francophone et auteur français. En fait, tout porte à croire que la distinction se fait dans l'esprit spontanément, sans exercice conceptuel, par rapport à l'univers culturel représenté dans une œuvre. L'accent tombe dans cette perspective sur l'idée de diversité. Plus un auteur utilisant le français comme langue d'expression a tendance à bâtir son œuvre autour de traditions culturelles autres que celles attribuées au monde occidental, ou encore à dépeindre des espaces culturels distincts de celui-ci, plus la possibilité qu'il soit considéré comme un auteur francophone, se renforce. C'est ce qui fait, par exemple, qu'un poète surréaliste et expérimentateur du langage comme Gherasim Luca, 
né en Roumanie, de langue maternelle roumaine, devenu français malgré lui et vers la fin de sa vie, mais écrivant en français la plus grande partie de son œuvre, a été adopté par la culture française, alors que Léon-Gontran Damas, né dans la Guyane française, ayant écrit l'intégralité de son œuvre en français et prêté sa voix aux Caraïbes, se voit étiqueter "poète francophone ». Comment définir un texte ou un film francophone sans s'enliser dans ce genre de distinctions artificielles ? Car, si rien ne semble s'opposer à l'emploi du mot « francophonie » pour désigner une réalité institutionnelle et linguistique, son implantation sur le plan culturel ne va pas de soi. À vrai dire, il n'y a pas d'objet culturel francophone puisque ce mot ne recouvre aucune détermination incontestable au-delà de l'idée trop vague de diversité culturelle. Aussi, pour échapper à cette impasse, faudrait-il peut-être employer le concept de discours francophone qui transcende toute démarcation entre des objets culturels concrets. Ce concept ouvre la possibilité de définir, sur le plan culturel, la francophonie non comme un inventaire d'œuvres, mais comme un laboratoire d'idées et de représentations. Le discours francophone se constitue, dans cette acception, en un univers de paroles et d'images qui se forment lorsque, dans une conjoncture historique donnée, à travers le français, deux ou plusieurs cultures différentes entrent en contact. Il va de soi que le discours francophone comporte un vaste répertoire topique, mais il n'en reste pas moins qu'il se construit selon un certain nombre de schémas qui déterminent sa typologie. De ce point de vue, une œuvre peut porter le discours francophone ou non, quelles que soient les conditions historiques et culturelles de sa production. Ainsi, étudier les œuvres relevant de la francophonie culturelle revient, en fin de compte, à y déceler la présence de ce discours, à rendre compte de ses configurations et de ses présupposés.

Quel discours nourrit donc la francophonie post-soviétique? Qu'est-ce qui s'énonce et s'imagine lorsque, après 1989 , se met en place la plateforme sociale où se produit l'interaction entre les porteurs de la culture de l'«autre Europe » et les représentants de la civilisation francophone occidentale? Autour de quels axes, par ailleurs, se constitue ce discours ? Quels en sont les invariants? Si l'on s'accorde pour distinguer, sur le plan de la francophonie culturelle, entre un discours postcolonial, un discours «beur», un discours «migrant» (au Québec), un discours «minoritaire» (dans les régions anglophones nord-américaines), etc., quelle serait la spécificité du discours francophone post-soviétique? Si l'on veut systématiser, trois grands thèmes semblent structurer cette parole qui résonne de plus en plus fort dans l'Europe francophone d'après l'effondrement du « Rideau de fer » : 
1. Le discours différentialiste ou de l'exotisme qui exploite au maximum l'écart entre les cultures des deux Europe. Il se fonde sur une théâtralisation de l'altérité, sur la quête et l'exhibition de l'insolite. Souvent, il joue la carte du mystère, voire du fantastique. Traditions, croyances, histoire, environnement naturel, comportements, tout peut devenir objet du discours différentialiste. L'accent est mis sur la représentation du pittoresque de l'« autre Europe », de ses lieux et modes de vie, ainsi que de quelques figures-stéréotypes comme le gitan, le malfaiteur, le mendiant ou «l'homme du peuple ».

2. Le discours dénonciateur qui évoque les aspects les plus sombres de la vie sous les régimes totalitaires de nature soviétique : la terreur, l'étranglement des libertés fondamentales, la précarité de la vie matérielle, etc. C'est essentiellement un discours mémoriel puisant dans les réminiscences alimentées par l'expérience personnelle ou bien dans le réservoir de la mémoire collective. Souvent, c'est la période de transition vers la société démocratiquement organisée qui prend le devant de la scène, notamment le chaos et le bouleversement qui s'installent à la chute des régimes communistes.

3. Le discours migrant qui dépeint les aléas propres à l'expérience transfrontalière, l'ensemble des mobiles, obstacles, déboires et franchissements qui la définissent. Ce discours prend pour objet différentes circonstances liées à l'insertion sociolinguistique de l'individu parti à la recherche d'une «meilleure vie ».

Ces trois discours se diffusent et se croisent dans l'espace public. Ils se reflètent particulièrement dans la littérature et le cinéma de langue française qui abonde de scénarios narratifs et de représentations où l'on reconnaît leur présence. Mais quelle est au juste la teneur de ces discours constitutifs de la francophonie post-soviétique et de quelle manière sont-ils incorporés aux œuvres? Telles sont les questions que se doit de prendre en charge l'étude de cette francophonie et pour lesquelles on peut déjà esquisser des éléments de réponse.

\section{Le discours littéraire et cinématographique francophone post-soviétique}

Le nombre des auteurs écrivant dans le contexte socioculturel post-soviétique et dont l'œuvre porte les discours de ce versant de la francophonie européenne, est considérable. On retrouve dans cette liste des noms proéminents comme ceux de Milan Kundera, Andreï Makine, Agota Kristof, Matein Visniec, Dumitru Tsepeneag, mais aussi des écrivains émergents ou de notoriété modérée comme Brina Svit, Marius Daniel Popescu, Dimitri Bortnikov, Valeri 
Afanassiev, Beata de Robien, Liliana Lazar, Rouja Lazarova, Maria Maïlat, Bessa Myftiu Velibor Čolić ou encore Katrina Kalda.

Le cinéma francophone post-soviétique s'individualise par l'abondance des coproductions qui mettent en scène les multiples aspects des interactions entre les deux Europe qui voient s'effacer, en 1989, les frontières qui les séparaient. Parmi les œuvres plus largement connues on peut mentionner La double vie de Véronique (1991) et Trois couleurs : blanc (1994) de Krzysztof Kieślowski, J'ai pas sommeil (1994) de Claire Denis, Code inconnu (2000) de Michael Haneke, Tirana année zéro (2001) de Fatmir Koçi, Je vous trouve très beau (2005) de Isabelle Mergault, Transylvania (2006) de Tony Gatlif, Le silence de Lorna (2008) par les frères Dardenne, Le Concert (2009) de Radu Mihaileanu, Indigène d'Eurasie (2010) de Šarūnas Bartas, Une trop bruyante solitude (2011) de Vera Caïs, L'étranger (2012) de Niki Iliev, Marussia (2014) de Eva Pervolovici. Encore insuffisamment exploré, cet ensemble croissant d'œuvres cinématographiques produites ou coproduites en France, Suisse ou Belgique a fait récemment l'objet dans le monde académique anglo-saxon d'une série d'études à inclure dans toute bibliographie portant sur la francophonie post-soviétique: The New European Cinema Redrawing the Map (Rosalind Galt, 2006), European cinema after 1989 : Cultural Identity and Transnational Production (Luisa Rivi, 2007), European Cinema in Motion: Migrant and Diasporic Film in Contemporary Europe (Daniela Berghahn et Claudia Sternberg, 2010), Screening Strangers: Migration and Diaspora in Contemporary European Cinema (Yosefa Loshitzky, 2010), European Cinema After the Wall: Screening East-West Mobility (Leen Engelen et Kris van Heuckelom, 2014). Toutes ces études reconnaissent à juste titre dans le cinéma européen contemporain les signes d'une mutation générée par la transformation socioculturelle et politique de l'Europe après 1989. Cependant, le domaine francophone n'y étant abordé que partiellement, il est légitime de chercher à rendre compte de la spécificité des productions nourries par la dynamique transculturelle mettant en contact l'espace ex-soviétique et la francité occidentale.

Sans prétendre que l'univers imaginaire et discursif d'un ensemble d'œuvres fondé, en somme, sur leur seule appartenance à une même branche de la francophonie européenne, soit réductible à une matrice unique, on ne saurait nier la propagation dont jouit un certain mode de représentation de la réalité en particulier. Il s'agit notamment de la prééminence d'une esthétique non réaliste, qui extrait du tableau composite de l'Europe post-1989, des traversées et des 
croisements qui s'y dessinent, le ferment d'une transgression du réel où les espaces et les formes, les perceptions et les souvenirs s'emboîtent et se mêlent prodigieusement pour offrir l'image d'un spectacle féerique, onirique ou carnavalesque. Quel que soit le registre adopté, du burlesque au pathétique, les œuvres littéraires et cinématographiques du versant post-soviétique de la francophonie européenne assujettissent souvent leur intrigue au jeu de toutes les interférences : des cultures, des langues et des milieux sociaux, du réel et de l'imaginaire, du présent et du passé, etc.

Ce type de représentation nourrit, par exemple, le roman Hotel Europa (1996) de l'écrivain d'expression roumaine et française Tsepeneag, qui suit l'itinéraire picaresque de Ion, de Bucarest à Paris, à travers une Europe en pleine métamorphose après la chute du mur de Berlin. Le récit de ce voyage parsemé de péripéties et de mésaventures, ponctué de fantasmes et de désenchantements, s'avère être une allégorie de la rencontre, sous l'effet de l'immigration, entre les deux Europe longtemps séparées par le «Rideau de fer». Il arrive lors de cette rencontre que des mythes s'ébranlent, que des illusions se défassent, en même temps que se profile l'image d'un espace de la diversité culturelle où les contours ne cessent de révéler leurs porosités.

Si le roman de Tsepeneag place le parcours migratoire du protagoniste dans une atmosphère onirique, Un cœur de trop (2006) de Svit, écrivaine d'expression slovène et française, adopte comme stratégie pour symboliser le franchissement des seuils d'entrelacer réalité et fiction dans un récit où les événements s'étalent au gré des circonstances et du hasard. Un voyage de funérailles à Ljubljana, dans son pays d'origine, entraîne l'héroïne du roman dans une série de contingences qui coupe court à la stabilité de sa vie parisienne. Se propage ainsi, subrepticement, l'idée que la traversée des frontières et l'expérience transculturelle ouvrent la porte à l'imprévisible, à ce qui transgresse le réel connaissable.

Dans la même veine, enfin, est écrit le roman Repas de morts (2011) de Bortnikov, écrivain russe vivant en France. Le héros, un immigrant russe quelque peu névrosé, vit du côté du Père Lachaise, dans une chambre où il donne libre cours à ses souvenirs et à ses hallucinations. S'animent ainsi, à travers ces réminiscences, des êtres et des lieux de son passé ténébreux pour former un tableau hétéroclite qui abrite aussi des fulgurances du présent parisien. Son récit haletant est celui d'une fuite, d'une suite de traversées précipitées d'espaces et de milieux sociaux dans un univers vacillant. 
Une image tout aussi bariolée et chimérique de la réalité qui se révèle après 1989 aux Européens assistant à cet avènement, se projette dans les œuvres cinématographiques émanées de la mouvance francophone post-soviétique. Tel est le cas, par exemple, de la production Trois couleurs : blanc (1994) de Kieślowski qui expose les aventures rocambolesques d'un immigrant polonais à Paris retourné clandestinement dans son pays d'origine, maintenant capitaliste. Sur les mêmes coordonnées de l'invraisemblable et du carnavalesque se déroule l'action du film Le concert (2009) réalisé par Mihaileanu. Andreï Filipov, ancien chef d'orchestre au Bolchoï, et plusieurs musiciens d'origine juive licenciés sous le régime de Brejnev, livrés pendant trente ans à des emplois de subsistance, se font passer pour des membres du fameux orchestre pour venir jouer au Théâtre du Châtelet à Paris. Enfin, plus récemment, la production Marussia (2015) réalisée par Pervolovici suit, dans un registre tantôt réaliste tantôt merveilleux, l'errance parisienne d'une maman russe et de sa fille de six ans.

Ce ne sont là, bien entendu, que des indices d'une certaine contiguïté qui semble s'établir entre les productions littéraires et cinématographiques de la francophonie post-soviétique. Le regard qu'elles jettent sur la réalité européenne contemporaine, sombre ou enjoué, paraît se dérober sans cesse à la fixité. Il glisse plutôt à la surface d'un monde fait de diversité et de mutations pour n'en retenir que le spectacle étourdissant et baroque. Sans doute, cet ensemble reste-t-il à explorer, à être reconnu éventuellement comme une parcelle à part entière de la francophonie européenne et peut-être même comme corrélat d'une vision émanée du mondialisme contemporain.

\section{Bibliographie}

Bortnikov, Dimitri. Repas de morts. Paris : Allia, 2011.

Berghahn, Daniela et Claudia Sternberg. European Cinema in Motion : Migrant and Diasporic Film in Contemporary Europe. New York : Palgrave MacMillan, 2010.

Engelen, Leen et Kris van Heuckelom. European Cinema After the Wall : Screening East-West Mobility. Lanham : Rowman \& Littlefield, 2014.

Galt, Rosalind. The New European Cinema - Redrawing the Map. New York : Columbia UP, 2006. 
Gretchanaia, Elena, Alexandre Stroev et Catherine Viollet, dir. La francophonie européenne $X V I I I^{e}-X I X^{e}$ siècles. Perspectives littéraires, historiques et culturelles. Bruxelles : Lang, 2012.

Loshitzky, Yosefa. Screening Strangers : Migration and Diaspora in Contemporary European Cinema. Bloomington : Indiana UP, 2010.

Moussakova, Svetla, dir. Nouveaux visages de la francophonie en Europe, numéro spécial de la revue Les Cahiers européens de la Sorbonne Nouvelle., 2008.

Oktapoda-Lu, Efstratia, dir. Francophonie et multiculturalisme dans les Balkans. Paris: Publisud, 2006.

--- et Vassiliki Lalagiann, dir. La francophonie dans les Balkans. Les voix des femmes. Paris : Publisud, 2005.

Milosz, Czeslaw. Une autre Europe. Paris : Gallimard 1964.

Nowicki, Joanna et Catherine Mayaux, dir. L'autre francophonie. Paris : Champion, 2012.

Rivi, Luisa. European Cinema After 1989: Cultural Identity and Transnational Production. New York : Palgrave MacMillan, 2007.

Svit, Brina. Un cour de trop. Paris : Gallimard, 2006.

Tsepeneag, Dumitru. Hotel Europa. Paris : P.O.L, 1996.

\section{Films mentionnés}

Kieślowski, Krzysztof. Trois couleurs : blanc. 1994.

Mihaileanu, Radu. Le concert. 2009

Pervolovici, Eva. Marussia. 2015.

\section{NOTES}

1 Une autre Europe paraît en 1964 chez Gallimard, la version polonaise de l'essai ayant été publiée en 1959, toujours à Paris, sous le titre Rodzinna Europa (« l’Europe familière » en traduction littérale).

2 On peut mentionner, parmi les publications majeures qui s'inscrivent dans cette tendance, des titres tels $L a$ francophonie dans les Balkans/ Les voix des femmes; Francophonie et multiculturalisme dans les Balkans; Nouveaux visages de la francophonie en Europe ; L'autre francophonie; La francophonie européenne XVIII ${ }^{e}$-XIX ${ }^{e}$ siècles. Perspectives littéraires, historiques et culturelles. 